\title{
Network Topologies for dual band (UWB - transmit and Narrow Band- receive) Wireless Body Area Network
}

\author{
K.M.Silva \\ The University of Newcastle \\ Callaghan, NSW, Australia, \\ 2308. \\ kasunmaduranga.thotahewa \\ @uon.edu.au
}

\author{
M.R.Yuce \\ Monash University \\ Electrical and Computer \\ Systems Engineering \\ Clayton, VIC, Australia, 3800. \\ Mehmet.Yuce@monash.edu
}

\author{
J.Y.Khan \\ The University of Newcastle \\ Callaghan, NSW, Australia, \\ 2308 \\ Jamil.Khan@newcastle.ed \\ u.au
}

\begin{abstract}
This paper studies the performance of a Medium Access Control (MAC) protocol for an Impulse Radio Ultra Wide Band (IR-UWB) based Wireless Body Area Network (WBAN). Unlike conventional UWB wireless schemes, the proposed UWB based WBAN uses UWB pulses for transmission and a narrowband signal for receiving at the sensor node to achieve a low-power network. To investigate the performance of the UWB-WBAN system in a large environment like hospitals, two network topologies have been considered. One topology uses a router as an intermediate node to collect vital physiological information collected by on-body sensor nodes. In the second scenario, the sensors directly transmit the collected data to a central control node. Binary Pulse Position Modulation (BPPM) is used as the modulation scheme for UWB transmission and Frequency Shift Keying (FSK) is used for narrow band transmission. Another unique feature in the proposed WBAN network is that the sensor nodes utilize multiple pulses per bit during UWB transmission. Number of pulses sent per bit are dynamically varied according to received bit error rate and used to optimize wireless transmission, thus providing low-power consumption.
\end{abstract}

\section{Categories and Subject Descriptors}

C.2.1 [Computer-Communication Networks] Network Architecture and Design- Wireless communication

\section{General Terms}

Design, Performance

\section{Keywords}

UWB, Narrow Band, WBAN, IEEE 802.15.4

\section{INTRODUCTION}

Medical telemetry for remote patient monitoring has grown in popularity in recent years.

Permission to make digital or hard copies of all or part of this work for personal or classroom use is granted without fee provided that copies are not made or distributed for profit or commercial advantage and that copies bear this notice and the full citation on the first page. To copy otherwise, to republish, to post on servers or to redistribute to lists, requires prior specific permission and/or a fee.

BODYNETS 2011, November 07-08, Beijing, People's Republic of China Copyright (c) 2012 ICST 978-1-936968-29-9

DOI 10.4108/icst.bodynets.2011.247002
Wireless Body Area Networks (WBANs) provide patients with mobility which enables them to involve in their day to day activities while their vital body parameters are monitored in a remote location. Various wireless standards which are compared in Table 1 are being used for WBAN [1]. Ultra Wide Band (UWB) based technology stands out from other technologies due to the ability to produce high bandwidth at a lower transmission power.

A task group (IEEE 804.15.6 TG6) [2] was formed in 2007 in order to develop a standard for the WBAN. In a WBAN, vital physiological parameters can be measured using several wearable and implantable sensors. In this paper wearable sensors that contain two different communication links, UWB-transmit and narrowbandreceive, are considered for WBAN applications. The proposed WBAN network is based on three types of nodes as depicted in Figure 1. This enables the implementation of a WBAN in a large environment such as in hospitals, medical and care centers.

Table 1. Wireless standards for wban [1]

\begin{tabular}{|c|c|c|c|c|}
\hline \multirow[b]{2}{*}{ Properties } & \multicolumn{4}{|c|}{ Standard } \\
\hline & $\begin{array}{c}\text { IEEE } \\
\text { 802.15.4 } \\
\text { Zigbee }\end{array}$ & $\begin{array}{c}I E E E \\
802.11 b \\
W L A N\end{array}$ & MICS & $\begin{array}{c}I E E E \\
802.15 . \\
4 a \\
U W B\end{array}$ \\
\hline Frequency & $2.4 \mathrm{GHz}$ & $2.4 \mathrm{GHz}$ & $\begin{array}{c}401-406 \\
\mathrm{MHz}\end{array}$ & $\begin{array}{c}3.1-10.6 \\
\text { GHz }\end{array}$ \\
\hline $\begin{array}{l}\text { Transmit } \\
\text { power }\end{array}$ & $0 \mathrm{dBm}$ & $16 \mathrm{dBm}$ & $-16 \mathrm{dBm}$ & $-41 \mathrm{dBm}$ \\
\hline $\begin{array}{l}\text { Channel } \\
\text { Bandwidth }\end{array}$ & $2 \mathrm{MHz}$ & $22 \mathrm{MHz}$ & $300 \mathrm{kHz}$ & $\begin{array}{c}500 \\
\mathrm{MHz}\end{array}$ \\
\hline Datarate & $250 \mathrm{kbps}$ & $11 \mathrm{Mbps}$ & $\begin{array}{c}200-800 \\
\text { kbps }\end{array}$ & $\begin{array}{c}850 \mathrm{kbps} \\
\text { up to } 20 \\
\text { Mbps }\end{array}$ \\
\hline Range & $0-10 \mathrm{~m}$ & $0-100 \mathrm{~m}$ & $0-10 \mathrm{~m}$ & $2 \mathrm{~m}$ \\
\hline
\end{tabular}

As depicted in Figure 1, sensor nodes gather vital medical parameters such as Electrocardiogram (ECG), Electroencephalogram (EEG) and body temperature, and then transmit those data either to an intermediate router node or to a coordinator node. Router nodes act as intermediate nodes which route data and control messages between the coordinator and sensor nodes. Router node also controls the radio resources such as pulses per bit value (described in Section 2) for each of its child sensor nodes. Coordinator acts as the central node which collects sensor data. It also acts as gateway for higher layer applications which interprets data. Alternatively sensor data can be sent directly to the coordinator without routers. The networks utilized are based on star topology which is preferred in most WBAN applications due to the 
simplicity of the network design [3]. The performance of the network scenarios will be investigated herein by comparing power consumption, throughput, and packet delay and packet loss ratio.

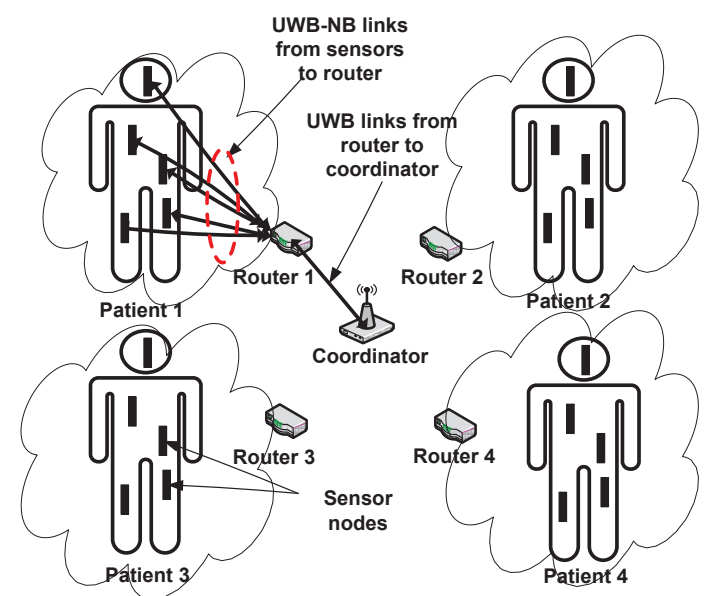

Figure 1. Implementation of UWB WBAN for a multi-human monitoring environment

Power consumption is considered to be a critical factor for battery powered on-body sensors. Although UWB transmitters are known to be as low power devices, UWB receivers are considered power hungry since they are designed to detect low level UWB signals [4] [5]. A transmit only UWB system is discussed in [4]. This system lacks the reliability of having a feedback path. It also has to occupy different receiver nodes for each patient, since different pulse repetitive frequencies are being used to identify different users. To avoid the use of power hungry UWB receiver and to increase the reliability of data delivery, a narrowband feedback path is suggested in the design of the sensor node. Having a feedback path also helps to make the network more coordinated, this enables us to reduce the multiple user interference (MUI) in an uncoordinated UWB network. In a system where both transmitter and the receiver use UWB, a turnaround time should be used to switch from transmit state to receive state. In other words, transmitter and receiver cannot operate at the same time due to the interference. Since we are using a narrow band receiver, it is possible to operate both transmitter and receiver simultaneously, hence reducing the packet delay.

A Medium Access Control (MAC) protocol has been designed to efficienlty provide the multi-access communication with the dualband WBAN architecture proposed here. The proposed MAC layer is based on the IEEE 802.15.4/4a [6][7] standards with adjustments done in order to facilitate a UWB transmission. Previous literature available for UWB based WBAN MAC focuss their attention on either contention based access [3] or time based access [8]. The suggested MAC protocol utilizes both time based and contention based data transmission abilities provided by above metioned standards. Because of the carrier-less nature of the UWB transmission, false alarms and miss detections are common. Those two types of errors have been taken into consideration in the simulation scenarios for the proposed WBAN system. Most of the available literature do not consider the precsence of multipaths in an indoor environment. Ricean fading channel [9] for UWB is considered in the presented simulation in order to simulate the effect of indoor propagation in a hospital environment. Simulation models for three types of WBAN nodes are developed in OPNET modeler [10], which is a commercially available simulation software. This paper consists of seven sections. Section 2 presents the modulation scheme and calculations with regard to false alarms and miss detection. Section 3 describes the MAC protocol used for the targetted WBAN architecture. Section 4 discusses the simulation scenario. Results are discussed in Section 5. Finally, Section 6 concludes the paper. Section 7 includes references.

\section{UWB-WBAN COMMUNICATION LINK}

\subsection{Modulation Scheme}

A Binary Pulse Position Modulation (BPPM) is used as the modulation scheme for the UWB transmission in the WBAN nodes. Energy contained in a bit is dependent on the number of pulses transmitted in that bit period [4]. This concept is used in the suggested MAC protocol in order to optimize the transmit power in the sensor nodes. Depending on the received signal strength, the parent node (router or coordinator) will send a feedback to the sensor node informing the number of pulses per bit to be generated. Note that this is achieved in the sensor node by varying the duration of the bit, not the pulse repetitive frequency. Hence the receiver node does not have to change the sampling frequency for each sensor node. It just keeps in track of the number of pulses per bit assigned to each sensor node. This can be achieved in the MAC layer. As a result, a single receiver node can be utilized for all the sensor nodes. Figure 2 depicts the use of two pulses per bit and three pulses per bit schemes for sending data bits. A short pulse (s (t)) is transmitted at a certain time slot (TS1 or TS2). If it is transmitted in TS1 it is considered as ' 1 ' and if it is transmitted in TS2 it is considered as ' 0 ' in a single pulse per bit scheme. This idea is extended to produce multiple pulses per bit schemes. A noncoherent receiver which detects the signal by comparing the energy received during a specific time slot against a threshold value is used as the receiver model (Figure 3) [11].

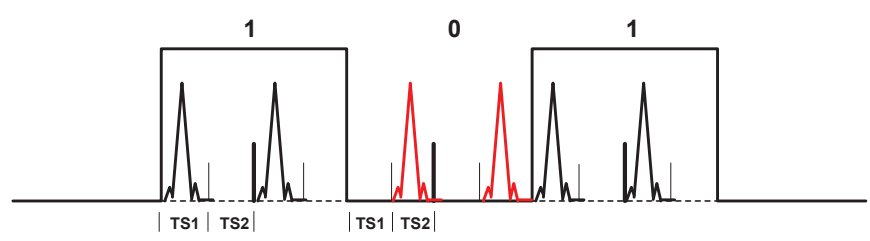

(a)

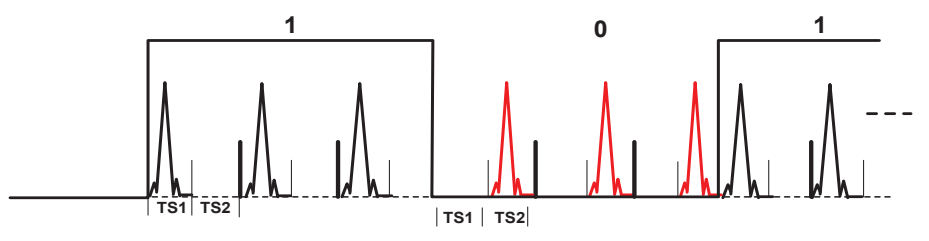

(b)

Figure 2. Pulse train for ' 1 ' ' 0 ' ' 1 ' bit pattern using two pulses per bit (a) and three pulses per bit (b) in BPPM

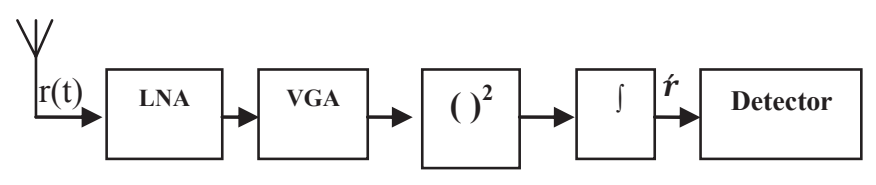

Figure 3. UWB receiver at the coordinator 
If the received signal energy at a certain time period is larger than a threshold value $(\alpha)$, a pulse is assumed to be present in that specific timeslot.

The input to the detector can be expressed as;

$$
\dot{r}=\left\{\begin{array}{cc}
n & \text { if there is no pulse present } \\
E+n & \text { in a time slot } \\
& \text { if there is a pulse present in } \\
\text { in a time slot }
\end{array}\right.
$$

where $\dot{r}$ is the input to the detector, ' $E$ ' is the energy of the detected pulse and $\mathrm{n}$ is a zero mean Gaussian random variable with variance $\sigma^{2}=\mathrm{N}_{0} / 2$, which is the contribution from Additive White Gaussian Noise (AWGN) to the detected signal energy. $\mathrm{N}_{0}$ is the power spectral density of the received noise. If hypothesis ' $p 1$ ' denotes a pulse not being sent at a given time slot and ' $\mathrm{p} 2$ ' denotes a pulse being sent in that time slot, the conditional probability density function of the random variable $r$ can be denoted as follows;

$$
\begin{gathered}
p(\dot{r} \mid p 1)=\frac{1}{\sqrt{2 \pi} \sigma} e^{-\frac{\dot{r}^{2}}{2 \sigma^{2}}}, \text { if a pulse is not transmitted } \\
p(\dot{r} \mid p 2)=\frac{1}{\sqrt{2 \pi} \sigma} e^{-(\dot{r}-E)^{2} / 2 \sigma^{2}}, \text { if a pulse is transmitted }
\end{gathered}
$$

Probability of errors is as follows;

When a pulse is transmitted,

$$
P_{e p 1}(\alpha)=P(\dot{r}<\alpha)=\frac{1}{\sqrt{2 \pi} \sigma} \int_{-\infty}^{\alpha} e^{-(\dot{r}-E)^{2} / 2 \sigma^{2}} d \dot{r}
$$

When a pulse is not transmitted

$$
P_{e p 2}(\alpha)=P(\dot{r}>\alpha)=\frac{1}{\sqrt{2 \pi} \sigma} \int_{\alpha}^{\infty} e^{-\dot{r}^{2} / 2 \sigma^{2}} d \dot{r}
$$

Assuming that sending of a pulse and not sending a pulse is equally probable probability of error;

$$
P_{e}(\alpha)=\frac{1}{2} P_{e p 1}(\alpha)+\frac{1}{2} P_{e p 2}(\alpha)
$$

By differentiation the optimum value of $\alpha\left(\alpha_{\text {opt }}\right)$ can be found. Probability of error can be shown as;

$$
P_{e}\left(\alpha_{o p t}\right)=Q\left(\sqrt{\frac{E}{2 N_{0}}}\right)
$$

where Q ( ) represents the Q function.

When multiple pulses per bit are sent it is assumed that a bit is erroneous when more than half the pulses sent per that bit are erroneous. If $\mathrm{N}$ number of pulses sent per bit, probability that a bit being erroneous can be obtained by

$$
P_{e_{b i t}}\left(\alpha_{o p t}\right)=1-\sum_{i=1}^{\left\lfloor\frac{N}{2}\right\rfloor}\left(\begin{array}{c}
N \\
i
\end{array}\right) p^{i}(1-p)^{N-i}
$$

, where $\mathrm{p}=P_{e}\left(\alpha_{\text {opt }}\right),\left(\begin{array}{c}N \\ i\end{array}\right)=\frac{N !}{i !(N-i) !}$ and $\lfloor x\rfloor$ is the inferior integer part of $\mathrm{x}$. Because of the coordinated nature of the network, it is assumed that the multiple user interference which happens because of pulse collisions is negligible. Modulation curves showing bit-error-rates (BERs) for different number of pulses per bit are simulated based on
(8) and presented in Figure 4. In the proposed WBAN system in this paper the parent node dynamically assigns the number of pulses per bit to be sent for the child node in order to obtain a pre given BER value at the receiver. A BER threshold of $10^{-4}$ is used in the simulation scenario for all sensor nodes because a good throughput can be obtained with this value while keeping the power consumption at the sensor nodes low.

\subsection{False Alarms and Miss detection}

As mentioned earlier, slotted Carrier Sense Multiple Access with Collision Avoidance (CSMA/CA) is used in the proposed MAC protocol for both sensor initialization for all sensors and data transmission of periodic sensors.

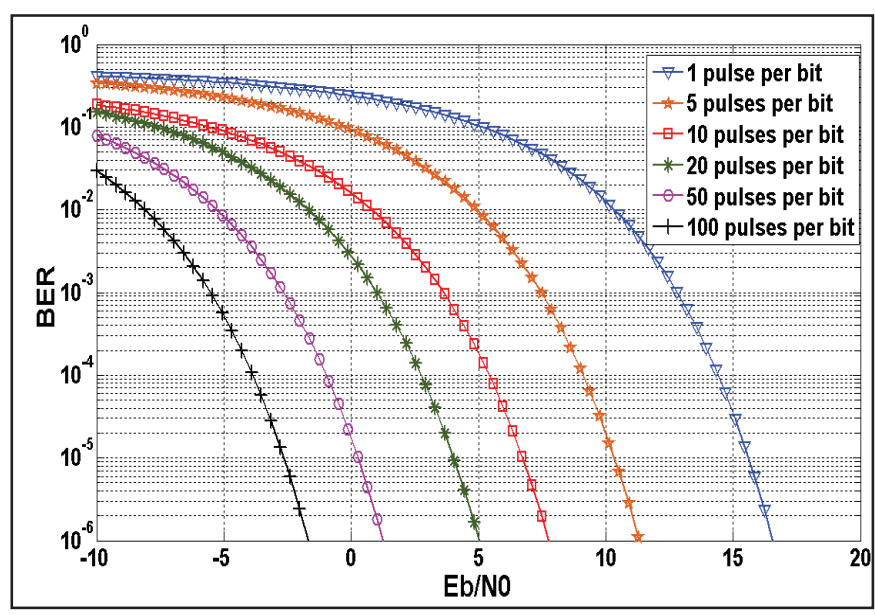

\section{Figure 4. Bit Error Rate (BER) Vs. Eb/No (dB) curves for different number of pulses per bit.}

A known preamble pattern of pulses are added at the beginning of a packet for the purpose of Clear Channel Assessment (CCA), which is vital in a contention based protocol. A False Alarm (FA) is described as the event where a busy channel (preamble is present) is detected, when an idle channel (no preamble present) is present. A Miss Detection (MD) occurs when an idle channel is detected at the presence of a busy channel. Because of the carrier less nature of UWB signals both types of erroneous detections are present. Let the event 'I' denotes an idle channel and event 'B' denotes a busy channel. Assuming ' $U$ ' as the decider for the presence of a preamble sequence and can be expressed as:

$$
U=\sum_{i=0}^{N_{B}} \dot{r}
$$

where $N_{B}$ is the number of preamble bits and $\dot{r}$ is the input to the detector. If Additive White Gaussian Noise (AWGN) is assumed at the detector, and since the time bandwidth product $(\Phi=$ Integration Time $\times$ Signal Bandwidth) at the detector for received signal in UWB transmission of indoor environment is large, it can be assumed that decision variable ' $U$ ' at the detector follows Gaussian distribution. If $\mathrm{Pf}$ denotes the probability of false alarms and $\mathrm{Pm}$ denotes the probability of miss detection, those probabilities can be derived as [12];

$$
\begin{gathered}
\mathrm{P}_{\mathrm{f}}=\mathrm{P}\left\{\mathrm{U}>\zeta_{\text {opt }} \mid \mathrm{I}\right\}=\mathrm{Q}\left(\frac{\alpha_{\text {opt }}-\mu_{U}}{\sqrt{4 \mathrm{~N} \cdot \mathrm{N}_{\mathrm{B} .} \Phi}}\right) \\
\mathrm{P}_{\mathrm{m}}=\mathrm{P}\left\{\mathrm{U}>\zeta_{\text {opt }} \mid \mathrm{B}\right\}=\mathrm{Q}\left(\frac{\alpha_{\text {opt }}-\mu_{U}-\psi}{\sqrt{4\left(\mathrm{~N} \cdot \mathrm{N}_{\mathrm{B}} \cdot \Phi+\psi \cdot \mathrm{N}_{\mathrm{B}}\right.}}\right)
\end{gathered}
$$


where $\mu_{U}$ is the mean of the decision variable ' $U$ ' in the event of 'I', Sopt is the threshold for the detection of preamble, $\psi$ is the $E_{b} / N_{0}$ of the received signal. These probabilities were taken into account at the CCA stage of the simulated MAC protocol.

\section{MAC PROTOCOL FOR DUAL-BAND WBAN}

Set of commonly monitored physiological parameters are shown in Table 2. A WBAN consists of two types of sensor nodes depending on their data transmission rate. EEG and ECG are continuously transmitting sensors which require high data transmission rate and high guarantee of delivery. Sensors which transmit heart rate and blood pressure are periodic sensors and do not require high data rate. A Beacon enabled super frame structure shown in Figure 5 is used in the suggested MAC protocol. This follows the frame structure of IEEE 802.15.4 standard [6]. A super frame is divided into 16 time slots. These time slots belong to either Contention Access Period (CAP) or Contention Free Period (CFP). CAP uses slotted CSMA/CA for the access of the channel. In the suggested MAC protocol continuously transmitting sensors are assigned to the Guaranteed Time Slots (GTS) in the CFP, in order to prioritize the delivery of data. Without loss of generality, a Super frame order (SO) [6] is chosen as 4 and Beacon Order (BO) [6] is chosen as 6 for the simulation.

Table 2. Commonly monitored medical parameters [4]

\begin{tabular}{|c|c|c|c|c|}
\hline $\begin{array}{c}\text { Medical } \\
\text { Parameter }\end{array}$ & $\begin{array}{c}\text { Transmit } \\
\text { Period }\end{array}$ & $\begin{array}{c}\text { Sampling } \\
\text { rate } \\
\text { (samples/sec) }\end{array}$ & $\begin{array}{c}\text { Bits per } \\
\text { sample }\end{array}$ & $\begin{array}{c}\text { Data } \\
\text { rate } \\
\text { (bps) }\end{array}$ \\
\hline ECG & Continuous & 300 & 12 & 3600 \\
\hline EEG & Continuous & 200 & 12 & 2400 \\
\hline Heart Beat & Every 1 sec & & & 100 \\
\hline $\begin{array}{c}\text { Oxygen } \\
\text { Saturation }\end{array}$ & Every 1 sec & & & 100 \\
\hline $\begin{array}{c}\text { Blood } \\
\text { Pressure }\end{array}$ & Every1 min & & & 12 \\
\hline \begin{tabular}{c} 
Temperature \\
\hline
\end{tabular} & Every1 min & & & 12 \\
\hline
\end{tabular}

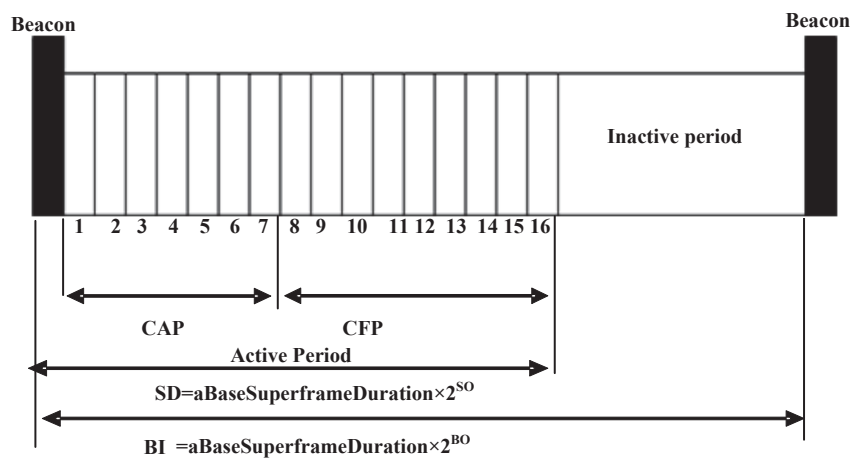

Figure 5. Super frame structure

Sensor nodes are initialized during the CAP. During the initialization, a pre defined pulses per bit value is used by sensor nodes to communicate with its parent node. This value is known to both sensor node and its parent node. A known bit pattern appended by a pre defined sensor address and sensor type flag (continuous or periodic) is sent as the initialization request. Sensor address consists of two parts; most significant eight bits will identify the patient to whom the sensor belongs to. Least significant eight bits will identify the sensor node. As a response to the initialization request, the parent node will send a positive or negative acknowledgement. If the acknowledgement is positive, it will assign pulses per bit value for that sensor depending on the power received at the receiver. If the request is from a continuous sensor node, the parent node will assign a time slot in CFP for that particular sensor. Data transmission occurs after the initialization.

\section{SIMULATION SCENARIO}

The simulation scenario is based on a $10 \mathrm{~m} \times 10 \mathrm{~m}$ hospital room where patients enter in a random manner (see Figure 1). Data rates used for sensors are taken from Table 2. To simulate the worst case scenario, data rates for the sensor nodes are calculated in order to represent the sensors that require highest data rates from each type. Which means, an effective data rate of 3600 bps (without over heads) are chosen for continuous sensors and a payload of 100 bits are sent every one second for periodic sensors.

A 433 MHZ ISM band with Frequency Shift Keying (FSK) is used for the narrow-band feedback link. Narrowband specifications are chosen according to a commercial narrowband receiver [13] and transmitter- TH72011 [14] datasheets. Two network topologies are simulated. One topology uses a router as an intermediate node between the sensor node and the coordinator node (Topology 1). In the other topology, sensor nodes directly coordinate with the coordinator (Topology 2). Cluster tree routing [15] which is used for Zigbee is implemented as the routing protocol. The motivation behind using a router as an intermediate node is to decentralize the coordination within the network by dividing it into sub networks. It also helps to optimize the power consumption in the sensor nodes. One time slot in the CFP is assigned per continuous sensor while periodic sensors contend using slotted CSMA/CA for transmission during CAP. Ricean fading channel for indoor propagation of UWB is used as the propagation mechanism [9], and the availability of Line of Sight (LOS) path is assumed. For a fair assessment of both topologies same numbers of sensors are simulated. Considering a realistic hospital scenario, five sensors are attached to each patient out of which one is a continuous sensor and others are periodic sensors [16]. A maximum of seven patients are assumed to enter the room during the simulation time. This number is decided based on the limitation of Guaranteed Time Slots (GTS) in topology 2. More sensor nodes can be assigned in topology 1 , since the data can be buffered at the router before sending to the coordinator node. The following performance related metrics are calculated for the simulation:

$$
\begin{array}{r}
\text { Throughput (\%) }=\frac{R(b p s)}{C(b p s)} \times 100 \% \\
P L=\frac{L}{S} \\
E\left(\mu \frac{J}{b i t}\right)=\left(\frac{\sum_{i=0}^{K}\left(I(A) \times V(V) \times T_{t x-r x}(\mathrm{sec})\right)}{\sum_{i=0}^{K} B}\right)
\end{array}
$$

$$
D=T 1-T 2
$$


where R (bps) is total data bit rate offered to the network by sensor nodes, $\mathrm{C}$ (bps) is the total network capacity, PL is the packet loss ratio, $\mathrm{L}$ is the total number of lost packets, $\mathrm{S}$ is the total number of sent packets, E $\left(\mu \frac{J}{b i t}\right)$ is consumed energy at a sensor node per useful data bit sent and acknowledgements received, I is the consumed current (A) of the sensor node, V (V) is the battery voltage, $\mathrm{T}_{\mathrm{tx}-\mathrm{rx}}(\mathrm{sec})$ is the sum of transmission time per a data packet and reception time for acknowledgments /control packets at sensor nodes, $\mathrm{B}$ is the total number of useful bits contained in above mentioned packets, $\mathrm{K}$ is the total number of packets sent, $\mathrm{D}$ is the packet acknowledgement delay for periodic traffic, T1 is the actual time at which the packet acknowledged and T2 is the time at which a packet enters the transmission queue.

Current consumptions used for simulation is shown in table 3. Battery voltage is used as $1.2 \mathrm{~V}$ which is the value for a typical standard battery.

Table 3. Current/power consumptions used for simulations

\begin{tabular}{|c|c|}
\hline Parameter & Current/ power consumption \\
\hline UWB transmit & $2 \mathrm{~mW} \mathrm{[5]}$ \\
\hline UWB receive & $16 \mathrm{~mA}[5]$ \\
\hline Narrowband receive & $6.5 \mathrm{~mA}[13]$ \\
\hline Sleep mode & $0.2 \mathrm{~mW}$ \\
\hline
\end{tabular}

\section{RESULTS}

From Figure 6 it can be seen that the packet loss ratio for the network topology which uses a router as the intermediate node is lesser than that of topology 2 where the sensor nodes directly coordinate with coordinator without an intermediate node. In topology 1 router nodes create a sub network with their child sensor nodes, hence the control of the system is more distributed than in topology 2. When sensor nodes directly send packets to the router, packets are buffered and sent to the coordinator. Packet losses in the network occur due to various reasons like bad channel conditions and collisions. When the network is divided into sub networks, comparatively small number of sensors contends for transmission in a shared medium. Hence the number of collisions is reduced. A router node can be kept close to the on-body sensors so that the received power level is better than a centrally placed coordinator node, providing better channel condition.

Figure 7 shows that the packet acknowledgement delay of periodic sensors in topology 1 is lower than that of topology 2 after the total number of active periodic sensors increases more than 20. Packet acknowledgment delay consists of the total time taken for queuing, medium access, inter frame space, packet transmission and acknowledgment reception. Medium access delay consists of two parts; 1. Time taken for back off procedure in slotted CSMA/CA 2. Time taken to finish Clear Channel Assessment (CCA). In topology 1 , only four periodic sensors per router have to contend for sending data. While in topology 2, all the periodic sensors contend for a single shared medium. As the number of sensors increases above a certain limit, the time delay that a sensor node is required to wait in order to send data through the shared medium will increase. Because of the high contention level in topology 2 after total periodic sensors increase above 20, it is observed from the simulation that both back off delay and time taken for CCA are higher. It is also observed that contention in the links between the routers and the coordinator in topology 1 is much lower than the contention level in the links between sensor nodes and coordinator in topology 2. From the results it is shown that even though topology 1 is a two hop network it is much more scalable than topology 2.With the introduction of narrow band feedback receiver, it is possible to do simultaneous transmission and reception at sensor nodes. Hence the time taken to turnaround from transmit state to receive state when using both UWB transmitter and receiver can be eradicated by using a narrowband receiver. This is shown in the simulation results since the packet acknowledgement delay for the system with narrow band feedback is lower than that of the system with both UWB transmitter and receiver in both topologies.

From Figure 8 it can be seen that throughput percentage which can be achieved for both continuous and periodic traffic in topology 1 is higher than that of topology 2. Router nodes in topology 1 offer channel resources only to their child nodes while in topology 2 , channel resources from coordinator node are shared between all the sensor nodes. The throughput percentage for periodic data decreases with increasing sensor nodes in all two topologies. This is because, as contention free traffic increases, timeslots available in super frame for contention based traffic decreases. The priority is given to continuous sensors which send data in the contention free period.

From Figure 9 it is seen that the consumed energy at sensor nodes per useful bit is higher in topology 2, which does not use an intermediate router. This power consumption value contains the power consumed for retransmissions as well. From the simulations it is observed that the number of retransmissions in topology 1 is less than that of topology 2 due to the distributed nature of the network. It should be noted that the router node is not required to be placed or attached to the body (like an on-body node) but it can be kept at close proximity to its child sensor nodes. Power consumption of an on-body sensor node is important for WBAN applications. In the topology 1 , which uses a router, the transmit power of the sensor node can be kept at a minimum in order to achieve a given bit error rate $\left(10^{-4}\right.$ in this simulation). Hence the number of pulses that needs to be sent is comparatively lower than a sensor node in topology 2. Since the signal strength at the receiver is fairly constant over short distance, the need of dynamically changing the number of pulses is lessen. Because of all these factors the sensor nodes in topology 1 consume less power than sensor nodes of topology 2. Power consumption is also compared between sensor nodes with narrow band receiver and UWB receiver. From the obtained results it is obvious that the use of narrow band receiver reduces the power consumption significantly in the sensor node.

\section{CONCLUSION}

In this paper we present a dual band- UWB transmit and narrowband receive WBAN system. The WBAN scheme is formed by two networks to enable remote monitoring of a multi-human body environment. A MAC protocol which is a modification of IEEE802.15.4/4a is suggested and studied for the proposed WBAN architecture. Furthermore, the MAC protocol is designed to dynamically vary the number of pulses per bit in UWB transmission according to the received signal condition. False alarms and miss detection are considered to improve the quality of communication. Performance is analyzed by comparing network metrics presented in the results. From the results it was observed that using a router as an intermediate node improves the data transmission over a WBAN. It is also shown that we can minimize the power consumption and packet delays for a UWB based WBAN sensor node using a narrow band receiver. 


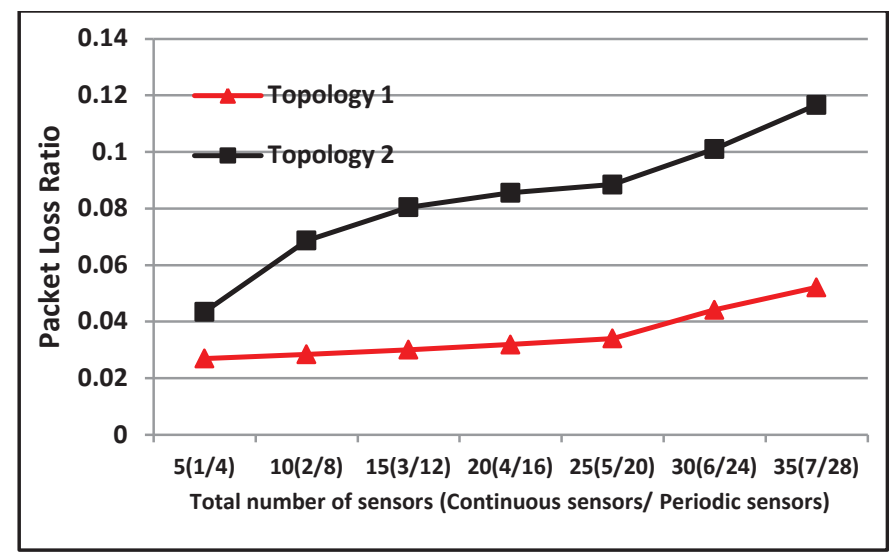

Figure 6. Variation of average Packet loss ratio with the increase of number pf sensors for each network topology.

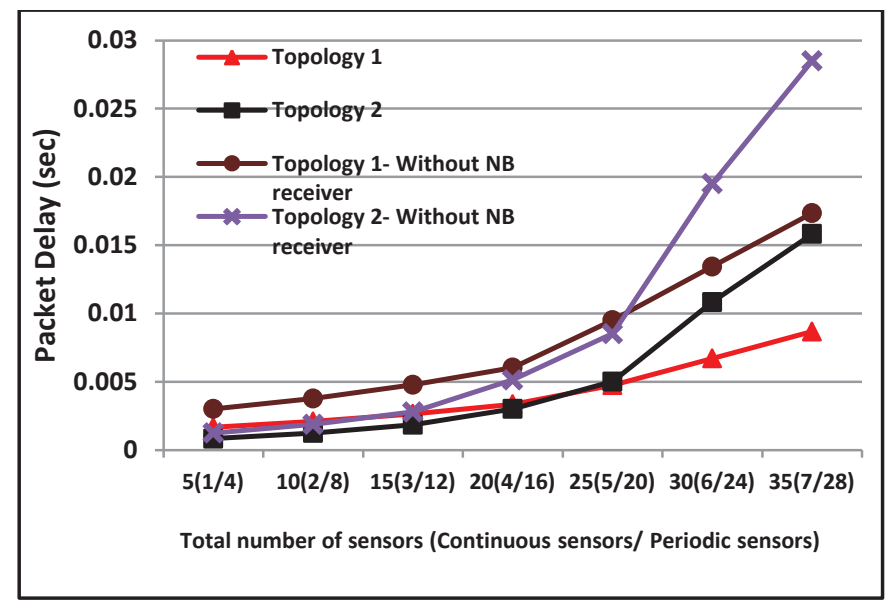

Figure 7. Variation of the average packet acknowledgement delay ( seconds) for periodic traffic with increasing number of sensor nodes for two topologies with and without narrow band feed back

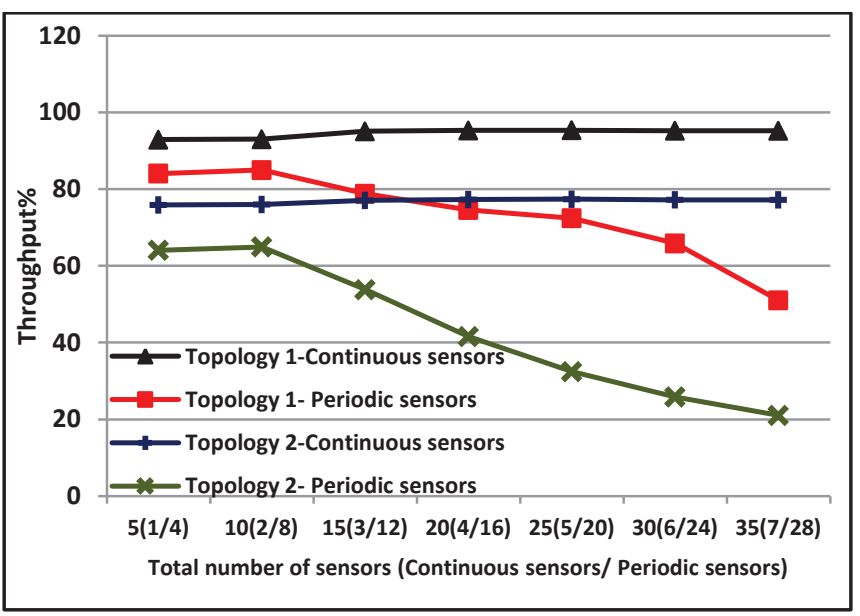

Figure 8. Variation of the percentage throughput for each sensor type in the two simulatated topologies.

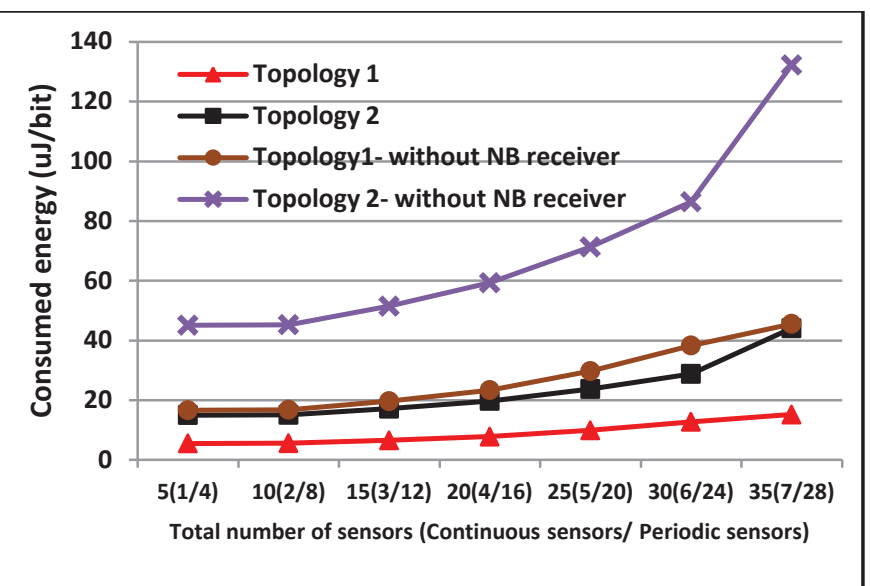

Figure 9. Variation of consumed energy by a sensor node per useful data bit (in $\mu \mathrm{J}$ ) with increasing number of sensor nodes for two topologies and for sensor nodes which use narrowband receivers and sensor nodes which use UWB receivers.

\section{REFERENCES}

[1] Yuce, M.R, 2010. Implementation of Wireless Body Area Networks for Healthcare Systems. Sensors \& Actuators: A. Physical, 162. (July. 2010), 116-129.

[2] http://www.ieee802.org/15/pub/TG6.html,.March 2010.

[3] Kynsijärvi, L., Goratti, L., Tesi, R., Iinatti, J. and Hämäläinen, M., 2010. Design and Performance of Contention Based MAC Protocols in WBAN for Medical ICT Using IR-UWB, IEEE 21st International Symposium on Personal, Indoor and Mobile Radio Communications, (Istanbul, Turkey, September.2010), 107-111.

[4] Keong, H.C. and Yuce, M.R., 2009. Analysis of a multiaccess scheme and asynchronous transmit-only UWB for wireless body area networks. 31st Annual International Conference of the IEEE EMBS. (Minnesota, USA, 3-6 Sept. 2009), 6906-6909.

[5] Yuce, M.R., Dissanayake, T.N. and Keong, H.C. Wideband Technology for Medical Detection and Monitoring. In Naik, G.R. ed. Recent Advances in Biomedical Engineering, ISBN: 978-953-307-004-9, InTech Publishers, 2009. 335-360.

[6] IEEE-802.15.4, 2006. "Part 15.4: Wireless Medium Access Control (MAC) and Physical Layer (PHY) Specifications for Low-Rate Wireless Personal Area Networks (LR-WPANs)." Standard, IEEE

[7] IEEE-802.15.4a, 2007. Part 15.4, Wireless Medium Access Control (MAC) and Physical Layer (PHY) Specifications for Low-Rate Wireless Personal Area Networks (LR-WPANs): Amendment to Add Alternate PHY. Standard, IEEE.

[8] Gopalan, S.A, Jong-Tae, P., 2010. Energy-efficient MAC protocols for wireless body area networks: Survey. International Congress on Ultra Modern Telecommunications and Control Systems and Workshops (ICUMT). (Budapest, Hungary,18-20 Oct. 2010), 739-744.

[9] Punnoose, R. J., Nikitin, P. V. and Stancil, D. D., 2000. Efficient Simulation of Ricean Fading within a Packet Simulator. IEEE Vehicular Technology Conference. 2, (Sept 2000), $764-767$.

[10] http://www.opnet.com/solutions/network_rd/modeler.html.

[11] Gerosa, A., Solda, S., Bevilacqua, A., Vogrig, D., Neviani, A., 2009. An Energy-Detector for Noncoherent ImpulseRadio UWB Receivers. IEEE Transactions on Circuits and Systems I: Regular Papers. 56, 5. (May 2009), 1030-1040. 
[12] Haapola, J., Rabbachin, A., Goratti, L., Pomalaza-Raez, C., Oppermann, I., 2009.Effect of Impulse Radio-Ultrawideband Based on Energy Collection on MAC Protocol Performance, IEEE Transactions on Vehicular Technology, vol.58, no.8, Oct. 2009, 4491-4506.

[13] http://www.melexis.com/Asset/TH71101-DataSheetDownloadLink-4794.aspx. 2011.

[14] http://www.melexis.com/Asset/TH72011-DataSheetDownloadLink-4803.aspx, 2011.
[15] Sun, J., Wang, Z., Wang, H., Zhang, X., 2007.Research on Routing Protocols Based on ZigBee Network, Third International Conference on Intelligent Information Hiding and Multimedia Signal Processing. 1. (26-28, Nov2007), 639642.

[16] Yuce, M. R., Keong, H.C. and Chae, M., 2009. Wideband communication for implantable and wearable systems. IEEE Transactions on Microwave Theory and Techniques. 57, 2. (October. 2009), 2597-2604. 